
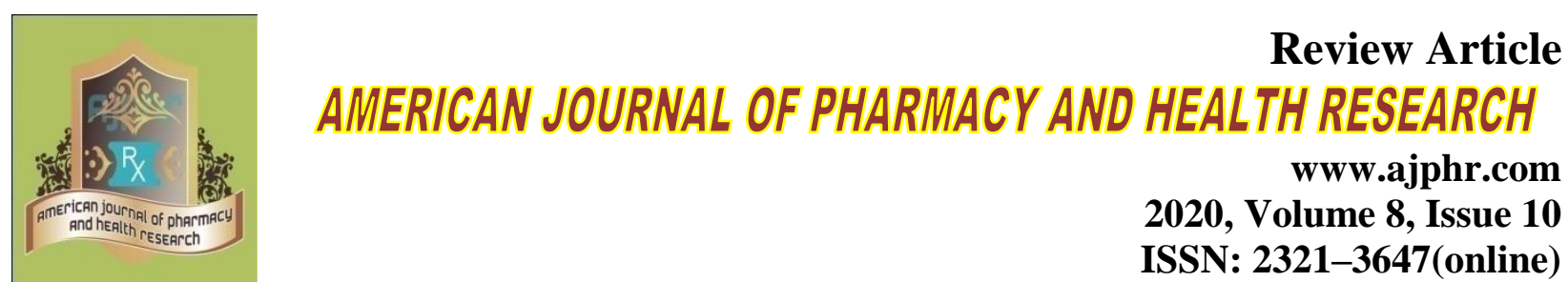

www.ajphr.com

2020, Volume 8, Issue 10

ISSN: 2321-3647(online)

\title{
Emerging Novel Drugs For Parkinson's Disease
}

\author{
Swathilakshmi S*, Vivek D, Aswini EV. \\ College of Pharmaceutical Sciences, Government Medical College, Pariyaram, Kannur- \\ 670503
}

\begin{abstract}
Parkinson's disease is the second most chronic neurodegenerative disorder, That impair motor skills, and cognitive performance. Most of the conventional therapies only focus on suppression of symptoms in the disease. But the emerging novel phytochemical compounds have significant effect on its Neuropsychopharmacological mechanisms. These compounds shows neuroprotective action and also decrease the oxidative stress, neuro-inflammatory pathogenesis of Parkinson's disease. Most of the adverse reactions of conventional treatment are reduced by novel compounds.
\end{abstract}

Keywords: Parkinson's disease, neuroprotective, neuroinflammatory, oxidative stress

*Corresponding Author Email: swathilakshmi1803@gmail.com

Received 10 September 2020, Accepted 28 September 2020

Please cite this article as: Swathilakshmi S et al., Emerging Novel Drugs For Parkinson's Disease. American Journal of Pharmacy \& Health Research 2020. 


\section{INTRODUCTION}

Parkinson's disease (PD) is a chronic, slowly progressive neurodegenerative disorder. It is the second most common age related neurodegenerative disease, after Alzheimer's. The disease was first described in 1817 by James Parkinson in his "An essay on the shaking Palsy". The disease is caused by the progressive loss of mesencephalic dopaminergic neurons in the substantia nigra pars compacta (SNpc) and subsequent depletion of dopamine in the striatum. Parkinson's disease can cause major disability and decline in quality of life. The cardinal physical signs include resting tremor, rigidity, bradykinesia and asymmetric onset.

The etiopathogenesis of Parkinson's disease is still not clear, but it may be caused by a combination of genetic and environmental factors which induce oxidative stress, mitochondrial dysfunction, $\alpha$ synuclein fibrillization and neuronal loss. Even though, PD is a multisystem disorder characterized by motor and non-motor (NM) symptoms.

\section{CLASSIFICATION OF PARKINSONISM}

\section{Primary (idiopathic) Parkinsonism}

Primary Parkinsonism is mainly due to degeneration of the dopaminergic neurons from the substantia nigra pars compacta, with the presence of Lewy bodies in the nerve cells. ${ }^{4}$

\section{Parkinson's disease}

There are two main forms of Parkinson's disease, familial and sporadic. About 5\% of those affected with PD are familial forms caused by gene mutations. The most common form is sporadic, about $95 \%$ cases, resulting from contributions of environmental and genetic susceptibility. The onset is usually in the late $50 \mathrm{~s} .^{5,6}$

\section{Familial PD}

Even though familial PD is rare, it has provided much information regarding the causes of this particular disease to the researchers. The pathogenesis of PD involves complex gene and geneenvironmental interactions. Mutations of autosomal dominant genes such as $\alpha$-synuclein, leucine rich repeat kinase-2 (LRRK2) and autosomal recessive genes such as parkin (PARK2), PINKI and DJ-I etc. ${ }^{1,6}$

\section{Sporadic PD}

Majority of the disease cases are sporadic forms, which involves the multiple neuronal systems. It involves the selective degeneration of bounded portions of the nervous system. Sporadic PD requires years to develop. The symptoms begin faintly, and worsen over time. ${ }^{7,8}$

\section{Secondary Parkinsonism}

\section{Drug induced Parkinsonism:}


Drug induced Parkinsonism (PID) is thought to second level following Parkinson disease (PD) in causing Parkinsonism, which accounts for up to $20 \%$ of the PD cases. The most common type of DIP is neuroleptics-induced Parkinsonism. It is strongly associated with the old antipsychotics, haloperidol, chlorpromazine, and pimozide. ${ }^{9}$

\section{Infectious:}

Post encephalic Parkinsonism (PEP) is distinctive among disorders with parkinsonian features because it has a unitary etiology caused by encephalitis lethargic.

\section{Metabolic:}

Metabolic syndrome is a common increasing public health problem that leads to many chronic diseases. Recent studies indicated that increased-oxidative stress is the heart and a common character of metabolism related disease.

\section{Structural:}

Structural changes of brain many leads to develop Parkinsonism. The major structural changes of brain arising mainly through Brain fever, Hydrocephalus, Trauma etc. ${ }^{10}$

\section{Toxins:}

Secondary Parkinsonism has been reported to occur due to large number of toxins including metals. The most important parkinsonian neurotoxins are 1-methy1-4-pheny1-1, 2, 3, 6tetrahydropyridine (MPTP), parquets, rotenone. ${ }^{11}$

\section{Vascular Parkinsonism:}

Vascular Parkinsonism (VP) commonly known as lower body Parkinsonism, resulting from progression of white matter lesions and lacunes as a result of thickening and narrowing arteries in the brain. Hypertension, DM, sleep apnoea and smoking are the vascular risk factors of VP. ${ }^{12}$

\section{Parkinson-plus syndromes:}

- Cortical-basal ganglionic degeneration (CBD)

It is the least common atypical Parkinsonism usually develops after age 60. There is no particular treatment for CBD. The symptoms include involuntary and jerk movements of a limb and speech problems.

- Dementia with Lewy bodies (DLB)

Dementia with Lewy bodies is the most common cause of dementia in the elderly. It results in the progressive intellectual and functional deterioration. People with DLB have a propensity of changes in ability to think, attention level or alertness and visual hallucinations.

- Multiple System Atrophy(MSA) 
Multiple system atrophy also called as Shy-Drager syndrome. It is a group of disorders in which one or more body system stops working. Major symptoms include bladder problems resulting in urgency, hesitancy or incontinence and orthostatic hypotension.

○ Progressive Supranuclear Palsy (PSP)

- The symptoms include forgetfulness, personality changes and loss of balance while walking that results in unexplained fails. The visual problems are also associated with PSP due to the paralysis of the muscles that move the eyeballs results in the inability to aim the eyes properly. ${ }^{13}$

\section{Hereditary degenerative disease}

- Autosomal-dominant cerebellar ataxias.

- Hallervorden-spate disease.

- Huntington's disease.

- Mitochondriopathies.

- Wilson's disease.

\section{PARKINSON'S DISEASE: SYMPTOMS}

\section{Motor symptoms}

- Tremor

Tremors are unilateral rhythmic oscillation of body part, which is happens at a frequency between 4 and $6 \mathrm{~Hz}$. There are further 20 kinds of tremor. The hallmark of PD is the resting tremor. It is the most common and easily identifiable symptoms of PD. Tremor usually starts in one hand, foot or leg and eventually affect both side of body. Resting tremor also occur in jaw, chin, lips, mouth or tongue. ${ }^{4}$

- Rigidity/hypertonia of gait

Rigidity refers the tightness or stiffness of limb or torso characterized by increased resistance usually accompanied by the "cogwheel" phenomenon. Rigidity might be related with pain. Painful shoulder is one of the most common manifestations of PD. ${ }^{15}, 16$

- Bradykinesia/kinesis

Bradykinesia refers slowness or absence of movement and it is the most significant and common characteristic clinical feature of PD. ${ }^{4}$ In PD, Bradykinesia is usually established by a loss of or mask like expression of the face (hypomania), decline in blinking of eyes and troubles with fine motor coordination. ${ }^{17}$

- Postural instability 
Postural instability includes the incapacity to maintain a steady, upright posture or to avoid a fall. It is usually a manifestation of late stages of PD. ${ }^{17}$

- Postural deformities

Postural deformities are resulted in bend neck and trunk posture and flexed elbows and knees, which are associated with rigidity. Flexed posture usually occurs later in the disease. $^{15}$

- Freezing

Freezing is a characteristic feature of PD. It referred to as motor block, which is a form of akinesia (loss of movement) and is one of the major disabling symptoms of PD. It mainly affects the legs during walking, but the arms and eyelids can also be involved. Freezing generally manifested as a sudden and transient inability to move. ${ }^{15}$

\section{Non motor symptoms}

Non motor symptoms are familiar feature of PD. These consist of:

- Autonomic dysfunction:

Autonomic dysfunction may be presenting feature of PD, even though it is related with MSA. Features include orthostatic hypotension, sweating dysfunction, sphincter dysfunction and erectile dysfunction. ${ }^{15}$

- Sleep disorders

- People with PD commonly experience sleep problems. The inability to stay asleep or secondary insomnia is more common than the inability to fall asleep

- Although in some case of PD, disruption of normal sleep wake cycle by taking catnaps throughout the day; doing this may lead to an inability to sleep at night

- Depression and anxiety

Depression and anxiety are comparatively common non motor symptoms of PD. Depression can vary in severity and can improve with PD treatment. Anxiety can be mild or severe and in some cases it requires medication.

- Sensational disturbances

The sensory symptoms such as olfactory dysfunction, paresthesia, akathisia, oral pain and genital pain are familiar but are not identified as Parkinsonian symptoms. ${ }^{17}$

\section{PATHOPHYSIOLOGY}

\section{Neuron loss}

Neuron loss is the hallmark of pathologic feature of PD, and it is essential for the diagnosis. It results the depletion of Dopamine (DA) neurons from the substantia nigra pars compacta (SNpc). 
At the time of death, PD patients have lost about $60 \%$ of their DA neurons even if it is affected mildly. Since the terminals of DA neurons are depleted, there is a reduction in DA uptake. This is coupled with several innate redundancies in DA terminals and DA receptors, which appears to allow the striated function to maintain without disruption or active compensation throughout the early phases of the neurodegenerative process.

Later rather larger lesions, the remaining DA terminals seen to increase the amount of neurotransmitter and delivered to extracellular fluid. This resulted in the net increase in the amount of DA released in response to terminal depolarization, a consequence of the transient disruption of homeostatic regulatory systems with in affected systems.

Apportion of the DA released appears to diffuse out of the synapse and into the extracellular space, whilst their action is prolonged due to the relative absence of high affinity DA uptake sites. These events allow the SNcp to continue to exert dopaminergic control over striatal cell function as long as some least number of DA terminals remains. The enlarged synthesis and release of DA might enhance the reactive metabolites produced from DA and thus add to the progression of the disease. Whilst there are various groups of dopaminergic neurons in the CNS, it is the depletion of DA cells in the SNcp that is assumed to account for all of the motor manifestations of PD. ${ }^{18}$

\section{Lewy Bodies}

Lewy Bodies (LBs) are another pathologic feature of PD, an eosinoplilic inclusion recognized within the neurons. Under histological stains, the Lewy Bodies contain an eosinoplilic core, and a surrounding pale halo. They are usually found within the cell soma, but also can be seen in neuritis or free in the extracellular space. They are normally observed in the brain regions showing the most neuron lost in PD including SN, locus coeruleus, the dorsal motor nucleus of the vagus, and the nucleus basalis of Meynert, but they are also seen in neocortex, diencephalon, and spinal cord and even in the peripheral autonomic ganglia.

Under the ultra-structural analysis, Lewy Bodies contain an electron dense granular core and a peripheral halo consisting of rapidly arranged filaments of 7 to $8 \mathrm{~nm}$ width. These filaments resemble neurofilament, and can be immunostained with antisera to neurofilament proteins. Immunostaining can be done using antibodies so as to identify both phosphorylated and nonphosphorylated epitopes. Presence of cellular proteins involved in protein degradation which includes ubiquityn and proteasome is another major characteristic feature of Lewy Bodies. The antigenic features of these cellular proteins are responsible for the cell to degrade the abnormal protein aggregate. Subsequent identification role of $\alpha$ - synuclein gene in the development of 
familial PD. It was revealed that $\alpha$-synuclein is a component of Lewy bodies. A protein known as Synphilin has been identified within the Lewy bodies in PD that interact with $\alpha$-synuclein.

The neurophysiology of PD is characterized exclusively by the depletion of dopaminergic neuron. 16 the degeneration of neuron and LB formations are found in non-adrenergic, serotonergic and cholinergic systems as well as in the cerebral cortex, olfactory bulb and autonomic nervous system. Disintegration of hippocampal structures and cholinergic cortical inputs induce high rate of dementia that accompanied PD. ${ }^{19,20}$

\section{TREATMENT}

\section{Pharmacologic therapy}

Monotherapy usually begins with a monoamine oxidase-B (MAO-B) inhibitor, or if the patient is physiologically young, a dopamine agonist.

When additional relief is needed, the addition of Levodopa (L-dopa) should be considered. With the development of motor fluctuations, addition of a catechol-O-methyltransferase (COMT) inhibitor should be considered to extend L-dopa duration of activity.

For management of L-dopa-induced dyskinesias, the addition of Amantadine should be considered.

\section{Anticholinergic Medication}

Anticholinergic drugs can be effective for tremor and dystonic features in some patients but rarely show substantial benefit for bradikinesia or other disabilities. They can be used as Monotherapy or in conjunction with other antiparkinsonian drugs. They differ little from each other in therapeutic potential or adverse effects.

Anticholinergic side effects include dry mouth, blurred vision, constipation, and urinary retention. More serious reactions include forgetfulness, sedation, depression, and anxiety. Patients with preexisting cognitive deficits and the elderly are at greater risk for central Anticholinergic side effects.

\section{Amantadine}

Amantadine is often effective for mild symptoms, especially tremor. It may also decrease dyskinesia at relatively high doses (400 mg/day).

Adverse effects include sedation, vivid dreams, dry mouth, depression, hallucinations, anxiety, dizziness, psychosis, and confusion. Livedo reticular is (a diffuse mottling of the skin in upper or lower extremities) is a common but reversible side effect.

Does should be reduced in patients with renal dysfunction.

\section{Levodopa and Carbidopa/Levodopa}


L-dopa, the most effective drug available, is the immediate precursor of dopamine. It crosses the blood-brain barrier, whereas dopamine does not. Ultimately, all PD patients will require L-dopa. The decision whether to start L-dopa as soon as the diagnosis is made or only when symptoms compromise social, occupational, or psychological well-being has generated controversy. In the CNS and elsewhere, L-dopa is converted by L-amino acid decarboxylase (L-AAD) to Dopamine. In the periphery, L-AAD can be blocked by administering carbidopa or Benserazide, which does not cross the blood -brain barrier. Carbidopa therefore increases the CNS penetration of exogenously administered L-dopa and decreases adverse effects (e.g. nausea, vomiting, cardiac arrhythmias, postural hypotension, vivid dreams) from peripheral L-dopa at $300 \mathrm{mg} /$ day (in divided doses) in combination with Carbidopa often achieves adequate relief of disability. The usual maximal dose of L-dopa is 800 to $1,000 \mathrm{mg} /$ day.

\section{Monamine oxidase inhibitor}

Monoamine Oxidase B InhibitorsAt therapeutic doses, Selegiline and Rasagiline are unlikely to induce a "cheese reaction" (hypertension, headache) unless excessive amounts of dietary tyramine (400 mg or greater) are ingested. However, concomitant MAO-B inhibitors with Meperidine and other selected analgesics is contraindicated. Selegiline and Rasagiline may be neuroprotective.

\section{Selegiline (deprenyl; Eldepryl)}

Seligiline an irreversible MAO-B inhibitor that blocks dopamine breakdown and can modestly extend the duration of action of L-dopa (up to 1 hour). If often permits reduction of L-dopa dose by as much as one -half. Metabolites of Selegiline are 1- methamphetamine and 1- amphetamine.

\section{Rasagiline}

Rasagiline is a MAO-B inhibitor, has similar effects as Selegiline in enhancing L-dopa effects and modest beneficial effect as Monotherapy. Early initiation is associated with better long-term outcomes.

\section{Catechol-O-Methyltranferase Inhibitors}

Tolcapone (Tasmar) and Entacapone (Comtan) are used only in conjunction with Carbidopa/Ldopa to prevent the peripheral conversion of L-dopa to dopamine (increasing the area under the curve of L-dopa by approximately 35\%). Thus, "on" time is increased by about 1 hour. These agents significantly decrease "off" time and decrease L-dopa requirements. Concomitant use of non-selective Mao inhibitors should be avoided to prevent inhibition of the pathways for normal catecholamine metabolism. 
COMT inhibition is more effective than controlled - release Carbidopa/L-dopa in providing consistant extension of effect and avoids the delay in time to maximal effect seen with controlledrelease L-dopa products.

The starting and recommended dose of Tolcapone is $100 \mathrm{mg}$ three times daily as an adjunct to Carbidopa/L-dopa. Its use is limited by the potential for fatal liver toxicity. Strict monitoring of liver function is required, and Tolcapone should be discontinued if liver function tests are above the upper limit of normal or any signs or symptoms suggestive of hepatic failure exist.

\section{Dopamine Agonists}

The ergot derivative bromocriptine (Parlodel) and the non- ergots pramipexole (Mirapex), rotigotine (Neupro), and ropinirole (Reequip) are beneficial adjuncts in patients with deteriorating response to L- Amantadine.

Amantadine is often effective for mild symptoms, especially tremor. It may also decrease dyskinesia at relatively high doses (400 mg/day).

Adverse effects include sedation, vivid dreams, dry mouth, depression, hallucinations, anxiety, dizziness, psychosis, and confusion. Livedo reticular is (a diffuse mottling of the skin in upper or lower extremities) is a common but reversible side effects.

\section{SAFINAMIDE}

Safinamide is an add on medicine that may be prescribed when individual taking levodopa and cardidopa have a breakthrough of Parkinson's symptoms that were previously under control. The most common side effects are trouble falling or staying asleep, nausea falls on uncontrolled involuntary movement. When you take levodopa, a chemical in your body called COMT makes parts of the drug useless. The drugs tolcapone and entacapone block COMT so brain can use levodopa more effectively which eases Parkinson's symptoms.

\section{BENZTROPINE}

These drugs restore the balance between two brain chemicals, dopamine and acetylcholine. That eases tremors and muscle stiffness in people with Parkinson's. But these medications can harm memory and thinking, epically in older people. Because of that doctors rarely prescribe them today.

\section{NOVEL DRUGS IN PARKINSON'S DISEASE}

Inbrija is an oral formulation indicated for the intermittent treatment OFF episodes in Parkinson's disease patients treated with a combination of carbidopa and levodopa.

\section{MECHANISAM OF ACTION}


- Inbrija is an oral inhalation drug that includes levodopa in dry powder formulation to be inhaled with the inhaler.

- The drug is a metabolic precursor of dopamine that crosses the blood-brain barrier and transforms into dopamine in the brain.

- Inbrija is available as white hypromellose capsule comprising $42 \mathrm{mg}$ of spray-dried

- Levodopa powder along with 1, 2-dipalmitoyl-Sn-glycero-3phosphocholine and sodium chloride.

\section{DOSAGE}

INBRIJA should be taken when symptoms of an OFF period start to return. The recommended dosage of INBRIJA is oral inhalation of the contents of two $42 \mathrm{mg}$ capsules (84mg) as needed, up to 5 times a day. The maximum dose per OFF period is $84 \mathrm{mg}$, and the maximum daily dosage is 420mg.INBRIJA has been shown to be effective only in combination with levodopa or carbidopa.

\section{ROUTE OF ADMINISTRATION}

Capsules are for inhalation only and should be used only with the inbrija inhaler; intended effect will not be obtained if capsules are swallowed. A complete dose (i.e., 84mg) is 2 capsules (42mg/capsules). ${ }^{29}$

\section{Physiochemical properties of liposome forms of DOPA and Dopamine}

Physiochemical properties of liposome forms of L-3, 4-dihyroxyphenylalanine (DOPA) and dopamine. .DOPA and dopamine encapsulated in liposomes have been obtained. This preparation can be used as drugs for PD. The level of encapsulation of the compound under study in liposomes and their partition coefficient has been determined. The kinetics of DOPA exit from the liposomes has been studied. ${ }^{26}$

\section{Apomorphine drug delivery system for Parkinson's disease}

The severity of PD is correlated to the degree of dopaminergic neuronal loss. Apomorphine has a similar chemical structure as the neurotransmitter dopamine and has been used for the treatment of advanced PD patients. In PD patients, apomorphine is normally administered subcutaniously with frequent injection because of the compounds extensive hepatic first pass metabolism .There is hence a large unmet need for alternative administered routes for apomorphine to improve patients compliance. The present review focuses on the research and development of alternative delivery of apomorphine, aiming to highlight the potential of non-invasive apomorphine therapy in PD, such as sublingual delivery and transdermal delivery. ${ }^{27}$

\section{History of conventional treatment of Parkinson's disease}


The discovery of dopamine as a neurotransmitter in the brain, its depletion in patients with the PD, and its replacement with levodopa therapy were major revolutionary events in the rise to effective therapy for patients with this disorder. This review describes these events and the persons who carried out this accomplishment. Their impact went beyond a single clinical entity of Parkinsonism, for its opened up the beginning of a much better understanding of the role of dopamine in other neurogenic movement's disorders and also in many psychiatric diseases. ${ }^{28}$

\section{COX-2 in the neurogenerative process of PD}

The enzyme COX-2 responsible for the first committed step in the synthesis of several important mediators which are involved in both initiation and resolution of inflammation, and the subsequent generation of prostaglandin upon activation has been shown to participate in the neurodegenerative processes of variety of disease. This review look particular in the role of COX-2 in the pathogenesis of PD, involving the generation of PGs and the role of the two different parts of the cyclooxygenase -cyclooxygenase and peroxidase activity. ${ }^{30}$

\section{Anticholinergic drug and amantadine in the treatment of PD}

Anticolinergics and amantadine both result in mild to moderate improvement in PD. These drugs still have an important role in the treatment of disease despite the greater clinical effect of levodopa and dopamine agonist. Benefits can be obtained in all stages of the disease but it typically most noticeable early on when disability is less marked. Many neurologists utilize these drugs initially in hopes of delaying the need for levodopa and later to permit the use of lower dose of these agents because of increasing concern that early treatment of levodopa may create many of long-term problems faced in the later stages of disease. ${ }^{28}$

\section{Prodrugs for the treatment of PD}

Current PD therapy is essentially symptomatic, and L-dopa is treatment choice in more advanced stages of the disease. However, motor complication often developed after long-term treatment, and this point physician usually prescribes adjuvant therapy with other classes of anti parkinsonian prod rug, including dopamine agonist, catechol -o-methyl transferees or monoamine oxidase -B inhibitors. This review discusses the evidence of progress in PD therapy, mainly focused on prod rug approach for treatment of this disorder

\section{Current pharmacotherapuetic treatment option in PD}

Treatment options for PD have greatly expanded in years .Pharmacological treatment such as levodopa, dopamine receptor agonist, anticholinergic medications, monoamine oxidase B inhibitor, and the catechol $-\mathrm{O}-$ methyl transferees inhibitor, remain the mainstay of therapeutic intervention and are reviewed. Additionally, the traditional and new roles for amantadine are 
explained. Despite the great efficacy of levodopa, "levodopa -sparing strategies" in early PD are embassies in order to delay the development of difficult-to -manage motor fluctuation and dyskinesia's.

\section{PHYTOCHEMICAL STUDIES ON PARKINSON'S DISEASE}

Potential phyto compound in drug development for PD: A pharmacoinformatic approach .PD is caused by human physiological function and in ranked as the second most common neurogenerative disorder. One of the prominent therapies currently available for PD is the use of dopamine agonist which mimic the natural action of dopamine in the brain and stimulate dopamine receptor directly. Currently, available pharmaceutical drugs provide only temporary relief of the disease . Phytocompounds have been identified as promising target research in the quest for new pharmaceutical compound as they can produce secondary metabolites with novel chemical structure .The drug development of PD has been analyzed using computational tool. ${ }^{24}$

\section{Pesticide use and Parkinson's disease}

A population-based case control study implicate the putative association between pesticide exposure and PD remains controversial. it identified all subjects who developed PD in Olmsted country, Minnesota, from 1976 through 1995, and matched them by age and sex to general population controls and assessed exposure to chemical products by telephone interview with cases, control, or their proxies. The association remained significant after adjustment for education for smoking. Analysis for the other six categories of industrial and household chemicals was all non signified.This population based study suggests a link between pesticides used and PD that is restricted to men. Pesticides ay interact with other genetic or no genetic factors that are different in men and women. ${ }^{25}$

\section{Hydroalcholic extract of Achyranthus aspera on haloperidol-induced PD in wistar rats}

The motor coordination of haloperidol-treated animals was evaluated by means of rotarod and hang test. Dopamine and 3,4-dihydroxyphenyl acetic acid were quantified using electrochemical detector and HPTLC. Antioxidant status were also assessed by estimating the levels of lipid peroxidation, superoxide dismutase, glutathione peroxidase, and reduced GSH to know the neurotoxicity of haloperidol. ${ }^{15}$

\section{Screening of antiparkinsonian activity of Allivum sativum in Swiss/mice}

Animals were treated with haloperidol $(2 \mathrm{mg})$ daily for a week to induce the PD.A combination of levodopa and carbidopa were used as a standard .Antiparkinsons effect of Allivum sativum has evaluated by catalepsy bar test, rotarod ,hang test and horizontal bar test. Statistical significance was tested using one way ANOVA followed by Bonferroin multiple comparison test .They 
concluded that Allivum sativum extract was found to decrease the duration of catalepsy when compared with the haloperidol group. The study showed that they ethanol extract of Allivum sativuim can be proposed to use in the treatment of PD. ${ }^{10}$

\section{Neuroprotective effect of Thuja orientalis in haloperidol induced animal model of PD}

Thuja orientalis, a common shrub widely found in India ,has showed neuroprotective effects against 6-hydroxy dopamine induced toxicity on SH-SY5Y cells. Adults male Spargue Dawely rats were divided into four groups and each group having 6 animal .The animal were treated with Thuja orientalis $(500 \mathrm{mg} / \mathrm{kg})$ Ibuprofen $(100 \mathrm{mg} / \mathrm{kg})$ and vitamin E $(35 \mathrm{mg} / \mathrm{kg})$ orally followed one hour after haloperidol $(2 \mathrm{mg} / \mathrm{kg}$ ) for 7 days. Behavioral parameter like motor activity ,rigidity, and catalepsy were assessed by using act photometer and common bar test .Statistical significance was assessed by using ANOVA and Kruscal-Wallis test followed by Dunn's multiple comparison test .A significant reduction in motor activity was observed in haloperidol treated experimental animals. $^{23}$

Antioxidant and antiparkinsonian activities of Brassica oleracea in haloperidol induced tardive dyskinesia

Hydroalcoholic extract of the plant was administered at the dose $1 \mathrm{mg} / \mathrm{kg}$ i.p. for 14 days, after the preliminary screening. Behavioral parameters were evaluated by measuring the locomotors activity using act photometer. Open field test and motor coordination by Rota rod and despair swim test and the cataleptic behavior by bar test. The biochemical parameters were also estimated. This study revealed that the hydro alcoholic extract of Brassica oleracea can be proposed to use in the treatment of PD. ${ }^{17}$

\section{Anti-cataleptic effect of phyllanthus amarus}

Ethanol extract of sample administered in Swiss albino mice .Haloperidol $1 \mathrm{mg} / \mathrm{kg}$ i.p. was administered to induce catalepsy in experimental animals. Behavioral parameters was assessed by using cataleptic bar test .Statistical significance was tested using ANOVA and the statistical comparison of group were performed with Tukey Kramer multiple comparison test using prism statistical package program. They concluded that the ethanol extract of Phyllanthus amarus have therapeutic role in Parkinsonism. ${ }^{18}$

\section{Antiparkinsonian activity of Mucuna pruriens and Aegle marmelose.}

Both the plants extract were prepared and treated. Benavioural parameters were assessed to measure the ant cataleptic effect of both the extract . Result showed a significance difference in the behavioral parameters between animals of Mucuba pruriens treated and combination treated groups. The plants have potential antiparkinsonian activity. ${ }^{19}$ 


\section{Antioxidant and anti-cataleptic effect of Nardostachys jatamansi}

Antioxidant and anti-cataleptic effects of the aqueous root extract of Nardostachys jatamasnsi in haloperidol induced catalepsy rat model. Haloperidol $(1 \mathrm{mg} / \mathrm{kg})$ was administered to induce catalepsy in male albino rats. Antioxidant potential was alone and in combination of extract in haloperidol $(1 \mathrm{mg} / \mathrm{kg})$ induced catalepsy rat model also assessed by estimating the level of lipid peroxidation, superoxide, dismutase, glutathione peroxidase etc. Result show that the aqueous extract of Nardostachys jatamasnsi reversed the haloperidol -induced catalepsy in rats and antioxidant potential has contributed to the reduction in the oxidative stress and catalepsy induced by haloperidol administration. ${ }^{22}$

\section{Antiparkinsonian activity of seed extract of Nelumbo nucifera}

A cataleptic effect of Nelumbo nucifera was evaluated by using metal bar test and block test. biochemical parameters such as thiobarbituric acid reactive substance; catalase; and superoxide dismutase, in brain were measured. The study discovered that Nelumbo nucifera treatment restored the levels of TBARS, catalase and SOD levels in haloperidol induced catalepsy in rats. ${ }^{21}$

\section{The antiparkinsonian activity of Uncaria rhynchophylla}

The anti-Parkinson's activity of aqueous extract of Uncaria rhynchophylla in 6 hydroxyl dopamine lesions rat model. The plant showed monoamine oxidase B inhibition and antioxidant activity. The pharmacological action of extract was evaluated by grip strength test, striatal dopamine levels, mitochondrial complex-I activity and glutathione level. They concluded that the Uncaria rhynchophylla aqueous extract exert a major neuroprotective effect and has a potential used for treatment of clinical Parkinsonism.

\section{Antioxidant and antiparkinsonian activity of Passiflora incarnate}

Antioxidant and antiparkinsonian activity of butane extract of Passiflora incarnate Linn leaves Antioxidant activity of BEPI was studied using DPPH scavenging assay and H2O2 scavenging assay. The antiparkinsonian activity of BEPI was studied using haloperidol-induced catalepsy and tacrine induced jaw movements. Haloperidol was administered to induce catalepsy in the experimental animals. The duration of catalepsy was measured at 0,30,60,90,120 and 150min after haloperidol administration using bar test. The study demonstrate that, BEPI possessed antiparkinsonian as well as antioxidant activity which may be effective for the relief of PD. ${ }^{20}$

\section{Antiparkinsonian activity of Ficus religiosa}

Antiparkinsonian activity of petroleum ether extract of Ficus religiosa leaves in haloperidol and 6-hydroxy dopamine induced animal model. Effect of Ficus religiosa were evaluated using various in vivo behavioral parameter like muscle rigidity and locomotion activity and neurochemical 
parameter in rats. Animal treated with 6-OHDA showed significant increase in the lipid peroxidation level and depleted superoxide dismutase, catalase, and reduced glutathione level. Study proves that Ficus religiosa treatment significantly attenuated the motor defects and also protected the brain from oxidative stress. ${ }^{21}$

\section{DISCUSSION}

PD is a chronic slowly progressive neurodegenerative disorder characterized by the loss of dopaminergic neurons of the SNpc. The second most common age-related neurodegenerative disease after Alzheimer's disease it was first described by James Parkinson in 1817.

Treatment of PD with the drug of choice, L-dopa, is limited only to the relief of symptoms. Existing classes of drugs such as DA agonist, Monoamine oxidase inhibitors, Catechol-OMethyltransferase inhibitor and anticholinergic agents may be used in the early stages of disease to relive PD symptoms. But none prevent the disease from progressing and show depilating side effects with prolonged use. Therefore, it is of utmost importance to develop new agents that shows or halt the rate of PD progression.

\section{CONCLUSION}

The conventional medicine and treatment, available today have proven ineffective in curing the multifunctional pathological mechanism of PD. Use of bioactive compound of mechanical plant on other hand has shown to possess the potential to modify or slow down the progress of PD. Identification of new bioactive compound becomes the need of the hour to develop new and effective drugs. It can concluded that the modern computational methods can strongly support and facilitate .The design of novel, effective and more potent inhibitor for PD with a better understanding of drug and receptor interactions. The computational tool $\mathrm{m}$ are the times save, avoid risk. Low cost and easy to detect the efficient molecules with that selected target.

\section{REFERENCES}

1. Harishankar P Y, Yun Li. The Development of Treatment for Parkinson's disease .Advances in Parkinson's disease.2015; 4:59-78.

2. Dipiro J T, Robert L T, Gary C Y, Curtis L. Pharmacotherapy: A physiological approach. $6^{\text {th }}$ edition .Newyork: MC.Graw Hill Companies;2008.chapter 57,Parkison's disease; $1075-89$

3. Tripathi K D. Essential of Medical Pharmacology. $7^{\text {th }}$ ed. New Delhi: Jaypee Brothers Medical Publishers; 2013.chapter 31,Antiparkinsonian Drugs;381-89.

4. Parkinson's disease Vs. Parkinsonism .National Parkinson foundation.2018 [cited 2018 Jan. 10]. Available from: www.parkinson.org. 
5. Braak H, Tredici K D, Rub U, de Vos R A, Ernst N H, Braak E. Staging of brain pathology related to sporadic PD. Neurobiology of aging.2003;24(2):197-97.

6. Zhang $\mathrm{P}$, Tian B. Metabolic syndrome: an important risk factor for PD.Hindawi.2014:1-7.

7. 7.Amos D Korczyn. Vascular Parkinsonism-characteristics, pathogenesis and treatment. Nat.Rev.Neurol. Advance online publication.2015 April;11(6):1-9.

8. Bhidayasiri R, Tarsy D. Parkinson's disease: Hoehn and Yahr Scale.Mov Disord.2012:4-5.

9. Standaert D G, SaintHilaire M H, Thomas CA. Parkinson's disease hand book [internet].2016 [cited Feb 3].available from: www.parkinsonsresource.org.

10. Zigmond M J, Burke R E. Neuropsychopharmacology[internet] $5^{\text {th }}$ ed.2002.chapter 123, Pathophysiology of Parkinson's Disease; 1781-93.

11. William Dauer, Serge Przedborki. Parkinson,s disease : Mechanism and Models .Neuron. 2003;39: 889-909.

12. Galvan A, Wichmann T. Pathophysiology of Parkinsonism.Clin.Neurophysiol.2008 July; 119:1459-74.

13. Hatano T, Hattori N. Ethiology and Pathogenesis of PD.Intech.2011.

14. Alexander G E. Biology of PD: Pathogenesis and pathophysiology of a multisystem neurodegenerative disorder .Dialogues in clinical neuroscience.2004;6(3):259-280.

15. Chitra V, Manasa K, Mythili A, Tamilanban T, Gayathri A. Effect of hydro alcoholic extract of Achyranthus aspera on haloperidol-induced PD in wistar rats.AJPCR.2017;10 (9):318-21.

16. Banu Z, Fathima J, Fathima A, Fathima S, Zohra S F, Sultana T. Physiochemical evaluation and Pharmacological screening of antiparkinsons activity of Alliuvum sativum in Swiss/albino mice.IOSR journal of pharmacy.2016 June;6:308-315.

17. Nagarjuna S, Arifullah,A M, Sanjeeva Kumar, Srinanth,K, Somasekar Reddy. Evaluation of antioxidant and antiparkinsons activities of Brassica oleraceae in haloperidol-induced tardive dyskinesia. Int.J .Green pharm.2015;9:143.

18. Deepa B, Anu E Joy. Effect of Phyllanthus amarus on haloperidol induced catalepsy in experimental animal models. IJABPT .2015 July; 6:199-203.

19. Bangalaes J O, Acharya S R. Anti-Parkinson activity of petroleum ether extract of Ficus religiosa leaves. Advances in pharmacological sciences.2015;4:1-10. 
20. Ingale S P, Kasture S B. Antioxidant and antiparkinsonian activity of Passiflora incarnate leaves. Orient pharm Ext Med. 2014;1-6.

21. Vardhan Reddy M V, Manmohan Singhal. Evaluation of anti-Parkinson activity of see extract to Nardostachys jatamansi in reversing haloperidol-induced catalepsy in rats.Int. J Pharma Bio Sci. 2014, April: 5(2):469-485.

22. Rasheed A S, Venkatraman S, Jayaveera K V, Fazil A M, Yasodha K J, Aleem M A. Evaluation of toxicological and antioxidant potential of Nardostachys jatamansi in reversing haloperidol induced catalepsy in rats.Int.J.Gen.Med .2010,May,10; 127-36.

23. 23 Saravanan R, Bavani E, Sakithbalan M. Neuroprotective effect of Thuja orientalis in haloperidol induced animal model of PD .IJPR,2016:308-15.

24. Vijayakumaar S, Manongar P. Review on potential phytocompound in drug development PD.A pharmacoinformatics in medicine unlocked .2016;5: 15-25.

25. Frigero R, Sanft K R, Grossardt B. Chemical exposure and PD:a population based case control study.Mov Discard. 2006 October;21(10):1688-92.

26. Borisova N V, Krioko , Shvets. .Phisico chemical properties of borate derivative of dopa, dopamine and their liposome form.Binoorg khim. 1998 Nov; 24(11):848-55.

27. Nrupa Borkar, Rene Holm. Challenges and trends in morphine drug delivery system for the treatment of PD. Asian Journal of pharmaceutical sciences. Nov 2018;3(6):507517.

28. Lang AE, Blair R D G. Anticholinergic Drug and amantadine in the treatment of PD. Handbook of experimental pharmacology.1989; 88:307-23.

29. Inbrija for the treatment of OFF episode in PD. Available from: https://www.clinicaltaialsarena.com/project/inbrija.

30. Stanley Fam M. The history of dopamine and levodopa in the treatment of PD. Movement disorder. Official journal of International Parkinson and Movement Disorder Society.2008;23(3):497-508.

\section{AJPHR is}

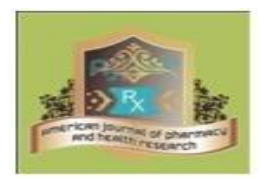

Peer-reviewed

monthly

Rapid publication

Submit your next manuscript at

editor@ajphr.com / editor.ajphr@gmail.com 